\title{
SISTEMAS DE CONTROL DE GESTIÓN Y DE MEDICIÓN DEL DESEMPEÑO: CONCEPTOS BÁSICOS COMO MARCO PARA LA INVESTIGACIÓN
}

\section{Management control and performance measurement systems: basic con- cepts as a framework for research}

\author{
Mónica Hernández Madrigal ${ }^{1}$
}

Recibido: 7/7/2016 • Aprobado: 10/10/2016

\begin{abstract}
Resumen
El propósito de este estudio consiste en analizar la forma en la que los sistemas de control de gestión y de medición del desempeño fomentan, impactan y mejoran el diagnóstico, la toma de decisiones y la ventaja competitiva basada en la perspectiva estratégica de las organizaciones. Además, se identifican los tipos y el diseño de diferentes sistemas que ofrecen información financiera y no financiera a diversos usuarios, considerando conceptos tales como contabilidad de gestión, control organizacional, sistemas de control de gestión y sistemas de control del desempeño. Este trabajo pretende contribuir a la literatura de la contabilidad de gestión al sentar las bases para un mejor entendimiento de las características de los sistemas de control de gestión y de medición del desempeño, integrando dichos conceptos en las organizaciones.
\end{abstract}

Palabras clave: sistemas de control de gestión; sistemas de medición del desempeño; contabilidad de gestión; estrategia.

\begin{abstract}
The purpose of this study is to analyze how management control and performance measurement systems encourage, impact and improve the diagnosis, the decision making process and the competitive advantage based on the strategic perspective of the organizations. The features and the design of various systems that offer financial and non-financial information to different users are identified, considering concepts such as management accounting, organizational control, management control systems and performance measurement systems. This work aims to contribute to the literature of management accounting laying the foundation for a better understanding of the characteristics of control and performance management systems, integrating these concepts into organizations.
\end{abstract}

Keywords: management control systems; performance measurement system; management accounting; strategy.

1. Profesora investigadora de la Universidad Autónoma de San Luis Potosí. Correo electrónico: hmadrigalmonica@uaslp.mx 


\section{El control organizacional y su enfoque en los resultados}

A través del tiempo, en la contabilidad de gestión se han involucrado tres actividades esenciales para las organizaciones: la planeación, el control y la toma de decisiones, es decir, el proceso de elegir entre dos o más alternativas o cursos de acción. En un sentido estricto, el Institute of Management Accounting (IMA, 2008) define la contabilidad de gestión como la profesión que vincula la toma de decisiones, la planeación y los sistemas de gestión de desempeño, con el fin de ofrecer experiencia en la información financiera y el control, que apoye la función de la administración en la formulación y la implementación de la estrategia organizacional, entendida esta última como el conjunto de acciones que ofrecen una ventaja competitiva a la organización frente a sus competidores y, en consecuencia, incrementan el valor agregado al cliente (Porter, 1980).

En este contexto, la contabilidad de gestión se fundamenta en la creencia de que la gestión de control es posible, importante y necesaria (Alvesson \& Kärreman, 2004). El control se entiende como la capacidad que se posee para mantener un dominio armónico sobre los bienes, las acciones, los acontecimientos y las personas, así como sobre todo aquello que puede ser un factor que altere, modifique, dificulte o impida alcanzar el logro de las metas que hayan sido establecidas, trazadas o señaladas en cualquier actividad humana, especialmente circunscritas al ámbito de las organizaciones actuales y de su futuro. De forma general, el propósito del control consiste en asegurarse de que alguien o algo que juega un rol importante sigue el guion previamente establecido (Macintosh \& Quattrone, 2010).

El concepto de control organizacional implica el proceso administrativo "diseñado para regular las actividades de los participantes de una organización y, por ende, sus fines" (Mills, 1983, p. 445), esto con el fin de "incrementar la probabilidad de que los individuos y grupos se comporten de forma que alcancen las metas organizacionales" (Flamholtz et al., 1985, p. 36). En este sentido, el control supone una constante revisión del diseño y de la adecuación permanente del proceso administrativo, acciones necesarias en la planeación estratégica, cuidando así su armonización con todos los elementos que conforman la organización e influyen en ella para la consecución de los fines propuestos.

Los mecanismos de control, definidos como el conjunto de planes, métodos, principios, parámetros y normas de verificación y evaluación de un sistema, se utilizan para motivar, supervisar, medir y sancionar las acciones de los gestores y los empleados (Macintosh \& Quattrone, 2010). Las prácticas de control de gestión dan lugar a las conexiones entre las diversas actividades de los miembros de la organización (Ahrens \& Chapman, 2007), influyendo en las metas, los objetivos y los fines de las mismas (Ahrens \& Mollona, 2007). Así, los resultados reales se comparan con los objetivos y las metas propuestas de forma participativa en la planeación.

Las herramientas o los medios básicos para el ejercicio de un adecuado control en las organizaciones son múltiples y, por naturaleza, variados. Algunos ejemplos de dichas herramientas son:

1. El empleo de métodos cuantitativos matemáticos, probabilísticos y estadísticos.

2. El uso de la tecnología ${ }^{2}$ para su aplicación en el control.

2. De acuerdo con Chenhall (2003), la tecnología se refiere a la forma como operan los procesos de las organizaciones, es decir, la transformación de insumos en resultados, incluyendo el hardware -máquinas y herramientas-, los materiales, la gente, el software y el conocimiento. En este sentido, las innovaciones técnicas en materia de los sistemas de control de gestión, tales como el costeo basado en actividades, el costeo por objetivos, el costeo en el ciclo de vida, el costeo de calidad, así como las innovaciones en la gestión del desempeño como el EVA, las medidas no financieras y los tableros de mando, constituyen una base para el desarrollo e implementación de nuevas estrategias y de tecnología avanzada (Chenhall \& Euske, 2007). 
3. El establecimiento de normas y lineamientos que orienten la actuación de los trabajadores. Estas normas pueden ser internas, es decir, debidamente consensuadas entre los miembros de la comunidad que participan en todos sus niveles, o externas, como resultado de disposiciones y normas que las correspondientes autoridades emiten en uso de sus atribuciones.

4. La aplicación de métodos psicológicos y sociológicos que induzcan o incentiven entre los miembros de la organización la eficiencia permanente, el trabajo en equipo, el concepto de mejora continua, de responsabilidad compartida y solidaria, el desarrollo de aptitudes personales y/o sociales, la pasión por el trabajo, la intuición y la visión del futuro. En este contexto, si bien dichos mecanismos de control son analíticamente distintos, no necesariamente se excluyen entre sí ni existe una forma dominante, sino más bien están unidos y apoyándose mutuamente (Alvesson \& Kärreman, 2004).

En conformidad con lo antes expuesto, las formas de control incluyen conceptos tales como la planeación, la observación, la supervisión, los resultados, los mecanismos, los procedimientos, los estándares, las evaluaciones, las estructuras, las reglas, las políticas, los presupuestos, las regulaciones, los reconocimientos, las prácticas, los cálculos y los incentivos de la acción individual y colectiva (Abernethy \& Stoeldwinder, 1995; Das \& Teng, 2001; Alvesson \& Kärreman, 2004; Dekker, 2004; Henri, 2006; Ahrens \& Chapman, 2007).

De acuerdo con Langfield-Smith (1997), las formas de control pueden ser clasificadas en formales e informales. Los controles formales incluyen reglas, procedimientos operativos estandarizados y sistemas presupuestarios, los cuales son retroalimentados y orientados financieramente, aseguran resultados específicos y suponen la supervisión, la medición y la toma de acciones correctivas. En contrapartida, los controles informales incluyen políticas no escritas derivadas de la cultura.

Por su parte, Malmi y Brown (2008) consideran cinco tipos de controles: de planeación, cibernéticos, de recompensa y compensación, administrativos y culturales. Estos controles se describen a continuación:

- La planeación establece las metas y las acciones para el futuro inmediato (planeación operativa) o a largo plazo (planeación estratégica).

- El control cibernético consiste en un sistema de apoyo a las decisiones que detecta variaciones desfavorables y modifica la situación que ha causado dichas desviaciones, tales como los presupuestos, las medidas financieras, las medidas no financieras e híbridos (medidas financieras y no financieras).

- Los controles de recompensa y compensación se basan en la motivación y el desempeño de los individuos y los grupos organizacionales al alcanzar una congruencia entre las metas personales y aquellas de la organización.

- Los controles administrativos dirigen el comportamiento de los empleados a través de la organización de grupos e individuos, especificando cómo deben ser desempeñadas las tareas y cuáles comportamientos deben ser realizados o no.

- Por último, los controles culturales regulan el comportamiento de los miembros de la organización basados en el conjunto de valores, creencias y normas sociales que comparten los empleados.

Finalmente, Merchant (1985) expone tres mecanismos de control: (1) control personal, el cual incluye los mecanismos que influyen en los miembros de la organización por medio de la alineación de sus objetivos con los objetivos de la misma; (2) control de acción, que se refiere a los mecanismos 
que influyen en los actores de la organización, señalando las acciones que deben seguir; y (3) control de resultados, que abarca los mecanismos que influyen en los miembros de la organización al medir los resultados de sus acciones.

Los tipos y los mecanismos de control con un objetivo en común, considerados de forma organizada e interrelacionada, se constituyen en la formación de un sistema de control de gestión.

\section{Los sistemas de control de gestión como base para la generación de información útil}

\subsection{Definición de un sistema de control de ges- tión: características y fines}

La esencia del pensamiento de sistemas concibe todo como partes interrelacionadas y reconoce que cada sistema es una parte de sistemas mayores y contiene subsistemas en sí mismo (Gray, 2002, p. 361). Todos los elementos de una organización se encuentran interrelacionados entre sí, siendo imposible aislar una entidad de sus circunstancias. En este sentido, la organización es un sistema interrelacionado con otros sistemas, subsistemas y ambientes de los cuales no puede prescindir, ignorar, aislarse o abstraerse al recibir de ellos, consciente o inconscientemente, influencias y consecuencias ${ }^{3}$ que, a la postre y en forma recíproca, la organización tendrá que responder, asumir o revertir, según sea el caso, prevaleciendo de forma permanente la incertidumbre y forzando la toma de decisiones a través de una planeación estratégica, flexible y oportuna.

La noción de sistema no se limita únicamente a la interrelación de sus distintos elementos dentro

3. Por ejemplo, fenómenos económicos, políticos y sociales; disposiciones legales, laborales, ambientales, sindicales, impositivas, de seguridad social o de calidad. del ambiente interno en que se desarrolla, sino que también exige una interacción y adaptación constantes con el entorno externo (Metzger, 2008). En este contexto, la contabilidad de gestión no solo se confina a los límites organizacionales, sino que va más allá al considerar las relaciones con otras entidades fundamentadas en procesos que fluyen de una organización a otra (Otley, 1994; Hopwood, 1996).

El diseño de los sistemas de gestión de control se apoya en el análisis de las distintas fuerzas económicas e institucionales (Boland et al., 2008). No existe un sistema de control de gestión único y adaptable a todas las organizaciones; en contraste, la elección de las técnicas de control dependerá de las circunstancias y de las condiciones específicas a su alrededor (Otley, 1999; Chenhall, 2003), tanto de forma interna como externa.

A pesar de que los términos de contabilidad de gestión, sistemas de contabilidad de gestión, control organizacional y sistemas de control de gestión a menudo se utilizan de forma indistinta, los sistemas de control de gestión abarcan tanto los sistemas de contabilidad de gestión como los controles financieros, no financieros, formales e informales (Chenhall, 2003).

La definición del sistema de control de gestión ha evolucionado. En el pasado, esta definición se enfocaba en la provisión de información formal y cuantificable financieramente; mientras que en la actualidad incluye información externa relacionada con mercados, clientes, competidores, información no financiera, información prospectiva, mecanismos de apoyo en las decisiones y controles informales y sociales (Chenhall, 2003). En consonancia, Otley (1999, p. 364) define un sistema de control de gestión como aquel que "ofrece información que se piensa es útil para los gestores en el desempeño de sus trabajos y ayuda a las organizaciones en el desarrollo y el mantenimiento de patrones de comportamiento viables". 
Con respecto al comportamiento de los empleados, Abernethy y Chua (1996, p. 573) definen un sistema de control organizacional como "una combinación de mecanismos de control diseñados e implementados por la gerencia para incrementar la probabilidad de que los actores organizacionales se comportarán de acuerdo con formas consistentes con los objetivos de la coalición organizacional dominante".

En síntesis, el sistema de control de gestión ha cambiado a través de los ańos, desde una perspectiva orientada hacia la provisión de información formal y financieramente cuantificable hacia una perspectiva de apoyo a la toma de decisiones gerenciales (Chenhall, 2003). La información que ofrecen los sistemas de control de gestión fluye hacia niveles inferiores con el objeto de que el área operativa tome mejores decisiones, apoyando así la integración de las operaciones con las prioridades estratégicas por parte de la alta gerencia (Chenhall \& Euske, 2007). Así, el involucramiento de los miembros que participan en el proceso, independientemente del nivel de mando que tengan asignado, impele a que todos sean actores responsables de los resultados esperados.

Entre los fines que persiguen los sistemas de control de gestión se identifican los siguientes: asegurar los intereses de los gestores, los accionistas y los stakeholders, con el objeto de cumplir con las expectativas externas (Ahrens \& Chapman, 2007; Boland et al., 2008); obtener una legitimidad institucional (Boland et al., 2008); fomentar la innovación de productos a largo plazo (Bisbe \& Otley, 2004); apoyar el cumplimiento de las leyes (Power, 2007); competir por recursos y eficiencia operativa (Boland et al., 2008); apoyar la gestión de riesgos (Boland et al., 2008); ayudar en la toma de decisiones para el logro de las metas organizacionales (Chenhall, 2003); favorecer la implementación, la supervisión y la reformulación de estrategias por medio del aprendizaje organizacional (Chenhall, 2003; Henri, 2006); facilitar los procesos fundamentales (core business) de la organización (Power, 2007); apoyar la integración de las actividades diarias de la organización con las prioridades estratégicas (Chenhall \& Euske, 2007); desarrollar un lenguaje único para el desarrollo de redes y la identificación de posibles interdependencias entre las partes de una organización y su entorno (Dekker, 2004; Chenhall \& Euske, 2007; Dekker, 2008); ofrecer información para la toma de decisiones estratégicas y operativas (Chenhall \& Euske, 2007) y fomentar la duración y la predicción entre redes organizacionales (Mouritsen \& Thrane, 2006).

De acuerdo con Power (2007), los sistemas de control de gestión (considerados por el autor como "tecnologías morales") pueden desarrollar dos grandes potenciales. El primero de ellos se refiere al logro de una mayor eficiencia y coordinación, lo que a su vez repercute en una actuación proactiva ante posibles crisis. El segundo consiste en una mayor sensibilidad sobre los asuntos relacionados con la responsabilidad social corporativa, la cual necesita ser apoyada por un sistema de control de gestión que promueva e institucionalice la toma de decisiones (Norris \& O’Dwyer, 2004).

En esta misma línea, considerando el ciclo de vida de un sistema de control, mientras que para algunos autores la etapa de crecimiento de una organización supone una mayor importancia de los sistemas de control de gestión por parte de la gerencia (Dávila, 2005), desde una perspectiva a largo plazo, el desarrollo de dichos sistemas muestra inicialmente una aceptación entusiasta por la organización, para después sufrir una disminución de la misma como consecuencia de la falta de beneficios que puede ofrecer a los usuarios y terminar con una etapa de renovación (Chenhall \& Euske, 2007). Basado en lo anterior, las organizaciones más grandes, más longevas y con mayor capital tienden a desarrollar sistemas de control de gestión de una forma más intensa.

Como consecuencia de la naturaleza dinámica de los sistemas de control de gestión (Chenhall, 2003), 
la organización no puede abstraerse del tiempo y de las circunstancias que la rodean y que influyen en ella; por el contrario, tiene que conocer, enfrentar y buscar las herramientas o los elementos que le permitan sobrevivir dentro de las nuevas condiciones de su ambiente, buscando e identificando las oportunidades que las mismas pueden brindarle y determinando las incertidumbres y las amenazas que pudieran presentarse. De tal modo, los sistemas de control de gestión permitirán encontrar respuestas y soluciones; cumplir los objetivos de eficiencia y eficacia operativa mediante la realización y la adecuación de sus productos y servicios; y minimizar los riesgos ya sea compartiéndolos, reduciéndolos, aceptándolos o eliminándolos; esto con el fin de satisfacer las expectativas de los distintos grupos de interés (stakeholders).

\subsection{Diseño de sistemas de control de gestión: innovación o isomorfismo}

El diseño de los sistemas de control de gestión supone una planeación orientada hacia el logro de resultados exitosos por parte de la organización. Para tal efecto, las organizaciones pueden optar ya sea por la innovación, o bien por la imitación de los sistemas de control de gestión de otras organizaciones exitosas. La primera alternativa considera un diseño basado en un proceso dinámico, influenciado por la resistencia y las presiones del entorno, tanto externas como internas. La segunda opción se fundamenta en un isomorfismo a partir de la imitación de las mejores prácticas corporativas de organizaciones exitosas (Boland et al., 2008).

Mientras que la innovación admite la necesidad de otorgar una mayor atención a las incertidumbres de carácter estratégico, como característica puntual de los sistemas de control interactivos (Bisbe \& Otley, 2004), el isomorfismo implica una tendencia por parte de las organizaciones a copiar "buenas organizaciones" y no "buenas soluciones"; es decir, "tienden a seguir el ejemplo de las organizaciones líderes aun cuando no tengan una idea exacta de los beneficios de los comportamientos que están copiando" (Boland et al., 2008, p. 904), con el objeto de alcanzar una mayor legitimidad por parte de los stakeholders poderosos (DiMaggio \& Powell, 1983).

No obstante, se decida por una u otra alternativa, en ambos casos no debe ignorarse que el cambio es inexorable y dinámico, que cualquier forma que se elija para el diseño del sistema de control de gestión (innovación o isomorfismo) no evita la renovación constante de estrategias, cuidando siempre de la eficiencia y de la eficacia de las operaciones. Por tanto, la perspectiva de los sistemas de control de gestión se define como dinámica (Henri, 2006; Chenhall \& Euske, 2007).

Las organizaciones se encuentran inmersas en un proceso dinámico de cambio que exige una renovación constante de sus estrategias. Según Otley (1999), hay cinco asuntos interrelacionados que deben ser considerados en el desarrollo y en el diseño de un marco de gestión del control organizacional:

(1) Objetivos: relacionando la definición de metas y sus mediciones, no únicamente financieras, sino también aquellas que logren cumplir con las aspiraciones de los stakeholders.

(2) Estrategias y planes: representando la codificación de los medios a través de los cuales se alcanzarán las metas y el balance entre los objetivos, los planes y las medidas de desempeño.

(3) Metas: reflejando el nivel y las medidas de desempeño, así como el seguimiento de ciertas prácticas de mejora continua, tomando en cuenta su eficiencia y eficacia.

(4) Estructura de incentivos y recompensas: incorporando la medición del desempeño con la gestión de recursos humanos, evitando en todo momento la visión de "corto plazo" de los trabajadores e incluyendo aspectos relacionados con el gobierno corporativo de las organizaciones. 
(5) Retroalimentación de información: considerando el aprendizaje organizacional a través de la experiencia, ya sea por medio de acciones correctivas o de una revisión completa de la estrategia de la organización.

Mientras que los tres primeros conceptos están relacionados con el diseño y la construcción de la planeación de presupuestos y de los sistemas de control, los dos últimos hacen referencia a las consecuencias de los mismos (Otley, 1999). En conclusión, según Otley (1999, p. 381), tres características son esenciales para el diseño de los sistemas de control de gestión: (1) el conocimiento de todas las actividades de la organización por los contadores de gestión; (2) la conexión de los sistemas de control con la estrategia de la organización y (3) el enfoque tanto en el ambiente interno de la organización como en su contexto externo.

Las organizaciones están cambiando su enfoque de poseer los negocios más atractivos a poseer aquellos en los cuales obtienen una ventaja competitiva (Buehler et al., 2008). Para tal efecto, las organizaciones pueden optar por el seguimiento de distintos tipos de estrategias: mercadotecnia superior, liderazgo en tecnología, minimización de costos, diferenciación, enfoque, mejora de tecnología existente, entre otras (Porter, 1980; Dávila, 2005). Con el objeto de lograr una ventaja competitiva y crear estrategias que generen valor, las organizaciones desarrollan recursos o capacidades distintivas, valiosas y difíciles de imitar, adoptar o sustituir por la competencia, como son el aprendizaje y la capacidad organizacional, la orientación al mercado y la innovación (Henri, 2006).

\subsection{Los sistemas interactivos de control de ges- tión y su relación con la estrategia corporativa}

Las organizaciones están cambiando su enfoque de poseer los negocios más atractivos a tener aquellos en los cuales obtienen una ventaja competitiva (Buehler et al., 2008). Para tal efecto, las organizaciones pueden optar por el seguimiento de distintos tipos de estrategias: mercadotecnia superior, liderazgo en tecnología, minimización de costos, diferenciación, enfoque, mejora de tecnología existente, entre otras (Porter, 1980; Dávila, 2005). Con el objeto de lograr una ventaja competitiva y crear estrategias que generen valor, las organizaciones desarrollan recursos o capacidades distintivas, valiosas y difíciles de imitar, adoptar o sustituir por la competencia, como son el aprendizaje y la capacidad organizacional, la orientación al mercado y la innovación (Henri, 2006).

El aprendizaje organizacional consiste en el desarrollo de conocimiento a partir de un análisis de las acciones pasadas y las futuras. La capacidad organizacional se enfoca en la habilidad de la organización para renovarse constantemente y tomar riesgos en los mercados y las áreas operativas. Por su parte, la orientación al mercado se fundamenta en el énfasis de la organización por cubrir las necesidades de sus clientes a largo plazo. Por último, la innovación se refiere a la creación de nuevas ideas, nuevos productos y procesos por parte de la organización (Henri, 2006).

La relación entre la estrategia y los sistemas de control de gestión debe ser examinada bajo las capacidades previamente señaladas (Henri, 2006). La estrategia incluye "los medios por los cuales los gestores pueden influir en la naturaleza del ambiente externo, en las tecnologías de la organización, en los planes estructurales, en la cultura de control y en los sistemas de control de gestión" (Chenhall, 2003, p. 150).

Los sistemas de control de gestión pueden enfocarse ya sea en su papel tradicional de retroalimentación, en apoyo a la implementación de la estrategia -uso de diagnóstico-, o bien en un papel más activo asociado con el fomento del diálogo y el soporte al surgimiento de nuevas estrategias -uso interactivo- (Simons, 1999; Henri, 2006). Así, mientras que el uso del diagnóstico (control mecánico) de 
los sistemas de control de gestión se enfoca en la supervisión y la recompensa del logro de las metas previamente establecidas, el uso interactivo (control orgánico) de los mismos supone el fomento de una mayor búsqueda de oportunidades y de aprendizaje organizacional (Henri, 2006).

Desde una perspectiva estratégica, los sistemas interactivos de control se describen como "sistemas de medición, utilizados para enfocar la atención en la información que cambia constantemente, la cual es considerada por la alta dirección como de una importancia estratégica" (Bisbe \& Otley, 2004, p. 711). Además de ser considerados sistemas de implementación de estrategias, los sistemas de control de gestión, incluyendo asuntos estratégicos y medidas de desempeño, representan el último paso del proceso de gestión estratégica y están basados en el trabajo en equipo y en las decisiones de la fuerza de trabajo (Henri, 2006; Chenhall, 2008).

A través de los sistemas de control estratégicos, la alta gerencia fomenta la participación de todos los miembros de la organización, enfocando su atención en las incertidumbres estratégicas, respondiendo a las oportunidades y a las amenazas percibidas y fomentando el aprendizaje, dando así lugar al flujo de diversas ideas y al surgimiento de nuevas estrategias (Bisbe \& Otley, 2004).

Teniendo como base las cuatro capacidades para lograr una ventaja competitiva -innovación, aprendizaje, orientación al mercado y capacidad organizacional-, así como las funciones que pueden desempeñar los sistemas de control de gestión -de diagnóstico e interactivo-, los resultados del estudio realizado por Henri (2006) señalan que los sistemas de medición del desempeño utilizados en forma interactiva contribuyen al desarrollo de dichas capacidades, a través del énfasis en las prioridades estratégicas, en el apoyo a la innovación, en la interacción con el ambiente y en el desarrollo del diálogo; sin embargo, los sistemas basados en la función de diagnóstico ejercen una presión negativa como consecuencia de las limitaciones impuestas con el objeto de asegurar el cumplimiento de los estándares establecidos, de los canales de comunicación demasiado estructurados y del conservatismo de la gerencia por "jugar seguro".

Es tal la importancia de los sistemas de control que los mismos influyen directamente en los cambios profundos ocurridos en las estrategias organizacionales (Bisbe \& Otley, 2004; Ahrens \& Mollona, 2007), esto como resultado de las presiones externas (Chenhall \& Euske, 2007), o bien apoyados sobre valores organizacionales compatibles (Bhimani, 2003).

No obstante la importancia de los sistemas de control muchas veces la dificultad de ponerlos en práctica reside, principalmente, en determinar cuáles actividades son importantes para proporcionar una seguridad razonable a los stakeholders, cómo dichas actividades deben ser llevadas a toda la organización, cómo estas pueden ayudar a fundir los resultados organizacionales (Ahrens \& Chapman, 2007) y cómo pueden ofrecer y apoyar el desarrollo de la ventaja competitiva de la organización.

El impacto de la estrategia sobre el desempeño de una organización está íntimamente ligado a los sistemas de control de gestión, siendo estos últimos elementos esenciales del ambiente interno de la misma (Bisbe \& Otley, 2004). La medición del desempeño, desde una perspectiva del ejercicio de las actividades inherentes a alguna actividad, se incluye como parte esencial de un sistema de control de gestión.

\section{Los sistemas de medición del desempeño basados en medidas financieras y no financieras}

Según Otley (1999, p. 364), "una organización que se desempeña bien es aquella que, en forma exitosa, alcanza sus objetivos; en otros términos, aquella 
que está implementando efectivamente una estrategia apropiada". Así, los objetivos representan la base para la elección de las medidas de desempeño, apoyados por la contabilidad de gestión y por los sistemas de control (Otley, 1999, p. 364).

Los sistemas de medición del desempeño constituyen un componente de los sistemas de control de gestión. Como se mencionó anteriormente, la función de ambos involucra desde su utilización, basada sobre todo en el diagnóstico, hasta una combinación que contempla tanto el uso de diagnóstico como el uso interactivo. El uso diagnóstico de los sistemas de medición del desempeño se define como un conjunto formalizado de procedimientos que utilizan la información para mantener o alterar patrones en una actividad organizacional (Henri, 2006). Cuando los gestores llevan a cabo un diagnóstico, las rutinas formales establecen guías para llevar a cabo las acciones correctivas (De Harlez \& Malagueño, 2016).

Por su parte, el uso interactivo de los sistemas de medición del desempeño supone un rol mayor, con el fin de expandir la búsqueda de oportunidades y de aprendizaje a través de toda la organización, generando nuevas ideas e iniciativas (Henri, 2006). $\mathrm{Al}$ respecto, el uso interactivo representa una fuerza opuesta de rutinas y procedimientos, promoviendo la innovación y la libertad de acciones (De Harlez \& Malagueño, 2016).

La combinación entre los sistemas de medición del desempeño basados en la función de diagnóstico y en la función interactiva a menudo crea conflicto o tensión, ya que, si bien los beneficios potenciales de la función interactiva pueden verse disminuidos como consecuencia del uso excesivo de diagnóstico que limita la innovación y la capacidad de arriesgarse, el diagnóstico también asegura los efectos positivos del uso interactivo en el logro de las cuatro capacidades estratégicas (Henri, 2006). Por tanto, las organizaciones inmersas en un ambiente de gran incertidumbre se beneficiarán aún más de la tensión provocada entre ambas funciones de los sistemas de control de gestión (Henri, 2006).

La medición del desempeño de una organización constituye un área de interés para la contabilidad de gestión. Empero, a menudo dicha actividad tiende a restringirse a las prácticas financieras de la organización basadas en aspectos económicos, dejando de lado las actividades internas de la misma y, en consecuencia, el diseńo del sistema de control de gestión (Otley, 1999). Para Koufteros, Verghese y Lucianetti (2014, p. 314): "un sistema de medición del desempeño puede también ser caracterizado como un sistema de control de gestión que incorpora un marco estructurado que especifica medidas clave de desempeño financieras y no financieras".

Para ilustrar lo antes expuesto, un sistema de medición del desempeño representa un conjunto de medidas -financieras o no financieras, internas o externas, a corto o largo plazo, ex post o ex anteutilizadas para cuantificar acciones (Henri, 2006) y como mecanismos que permiten a las organizaciones gestionar sus recursos de una forma más efectiva (Koufteros, Verghese, \& Lucianetti, 2014).

El uso de medidas no financieras para el control del desempeño de una organización no es un tema reciente, pues se remonta a los inicios a la década de los cincuenta. Sin embargo, en los últimos años se ha dado un enfoque particular al establecimiento de dichas medidas a partir de un aspecto estratégico de la organización (Norreklit, 2000). De acuerdo con Chua (2007), el hablar sobre estrategia implica, al mismo tiempo, tomar en cuenta la auditoría y la supervisión de sus efectos.

Las medidas financieras reflejan parcialmente la situación actual de la organización, ya que omiten el impacto de las acciones de hoy; en contraste, las medidas no financieras sí reflejan las acciones actuales de la administración a través de su seguimiento diario. En otras palabras, si bien las medidas no financieras no contienen más información que 
las financieras, sí brindan información incremental que ayuda a llevar a cabo mejores predicciones (Wiersma, 2008).

Los sistemas de medición del desempeño se integran a todos los procesos organizacionales. Incluso, en las últimas tres décadas muchas organizaciones han invertido grandes cantidades de capital, tiempo y esfuerzo desarrollando e implementando dichos sistemas (Koufteros, Verghese \& Lucianetti, 2014). El diseño y la implementación incluyen la formulación de las definiciones de las medidas de desempeño, la creación de datos de medición, la construcción de sistemas de información, el establecimiento de niveles de desempeño y la revisión de cada una de las medidas en particular y del sistema de desempeńo como un todo (Wouters \& Wilderom, 2008).

Ampliando lo anterior, durante el proceso de diseño e implementación se consideran todos los aspectos relacionados con el desempeño en términos cuantitativos (medidas financieras y no financieras), los intercambios entre medidas financieras y no financieras y la consideración de las interdependencias entre diferentes unidades organizacionales (Wouters \& Wilderom, 2008).

A manera de un mecanismo organizativo, las organizaciones pueden utilizar los sistemas de medición del desempeño para controlar el comportamiento organizacional y para promover la innovación y la renovación estratégica (Koufteros, Verghese \& Lucianetti, 2014). De acuerdo con el IMA (2008), el contador de gestión suministra el marco conceptual para convertir los datos en información; trabaja en equipo en todos los niveles de las actividades corporativas, lo que incluye su participación en la toma de decisiones estratégicas; contribuye al éxito de la organización por medio de la implementación de la estrategia; y cumple con el papel de asesor y socio estratégico de la organización a lo largo de todas las actividades de información que impactan en los clientes.
La medición del desempeño forma parte de la contabilidad de gestión contemporánea, fomentando el pensamiento estratégico de los gestores acerca de cómo sus actividades se entrelazan con otras partes de la organización (Chenhall, 2008). Los sistemas estratégicos de medición del desempeño son un subconjunto de los sistemas de medición del dese mpeño (Bisbe \& Malagueño, 2012).

Bisbe y Malagueño (2012) consideran que un sistema estratégico de medición del desempeño presenta las siguientes características: (i) la integración de una estrategia a largo plazo y de metas operativas; (ii) el establecimiento de medidas de desempeño desde múltiples perspectivas; (iii) el establecimiento de una secuencia de metas, medidas, objetivos y planes de acción para cada perspectiva y (iv) la presencia de relaciones entre metas y medidas de desempeño. Derivado de lo anterior, las capacidades de los sistemas de medición del desempeño son valiosas a medida que fomentan la ventaja competitiva (Peters, Wieder, Sutton \& Wakefield, 2016) y apoyan las estrategias de las organizaciones.

\section{Conclusiones}

Debido a la integración vertical de las organizaciones, a la diversidad de sus actividades y a la descentralización de las mismas en departamentos, las organizaciones demandan un sistema de contabilidad de gestión que suministre información a la alta dirección para la planeación, la coordinación y el desarrollo de estrategias a largo plazo. Con el fin de cubrir dicha necesidad, en las últimas décadas, la contabilidad de gestión ha evolucionado desde un enfoque orientado hacia el costeo intensivo de productos y servicios hacia la incorporación de conceptos estratégicos en las actividades de planeación y control organizacional.

En este cambio de enfoque se evoluciona de una contabilidad de costos relacionada con la valuación de inventarios a través de la acumulación de los 
mismos, con el fin de cumplir con los requerimientos de información al exterior, a una contabilidad de gestión enmarcada en el suministro de información dentro y fuera de los límites de la organización.

A diferencia de la contabilidad financiera tradicional, cuyas características de inflexibilidad y orientación hacia hechos pasados han restringido la visión prospectiva de la organización, la contabilidad de gestión supone como relevantes las actividades de planeación, de control, de gestión del desempeño y de toma de decisiones como apoyo en la formulación e implementación de la estrategia corporativa. En este contexto, los sistemas de control de gestión y de medición del desempeño constituyen temas esenciales de la contabilidad de gestión.

Los sistemas de control de gestión y los sistemas de medición del desempeño permiten efectuar cambios en la organización con el fin de mejorar su actuación dentro y fuera del entorno. Una vez terminado el diagnóstico, inicia el proceso de mejora basado en la comparación entre el desempeño real y el desempeño planeado, así como el establecimiento de nuevos estándares que sentarán las bases para la innovación y la promoción de nuevas acciones que incidan directamente en el éxito de la organización.

Asimismo, a pesar de que los sistemas de control de gestión y de medición del desempeño siguen utilizando medidas financieras enfocadas a largo plazo, las medidas de desempeño no financieras, tales como la innovación y el liderazgo de productos, la moral y la habilidad de los empleados, la lealtad del cliente, entre otras (Kaplan, 1984), empiezan a tomar un lugar relevante como indicadores del beneficio futuro de la organización.

Finalmente, los nuevos ambientes de producción requerirán no solo del desarrollo de nuevos procesos de control y de medición del desempeño, sino también de la reducción del énfasis en el corto plazo y del uso de indicadores no financieros que apoyen la generación de riqueza a largo plazo
(Johnson \& Kaplan, 1987). Por tanto, es importante que las organizaciones desarrollen ambos tipos de sistemas con el fin de medir, controlar y mejorar los resultados de forma holística. Dichas acciones pretenden fundamentalmente proteger el valor de los distintos grupos de interés y mejorar el desempeño organizacional a largo plazo, resaltando el papel preponderante que ejerce el control y el desempeño como parte integral de la contabilidad de gestión.

\section{Referencias}

Abernethy, M. A. \& Chua, W. (1996). Field study of control system 'Redesign': the impact of institutional process on strategic choice. Contemporary Accounting Research, 13(2), 569-606.

Abernethy, M. A. \& Stoeldiwnder, J. U. (1995). The role of professional control in the management of complex organizations. Accounting, Organizations and Society, 20(1), 1-17.

Ahrens, T. \& Chapman, C. S. (2007). Management accounting as practice. Accounting, Organizations and Society, 32(1-2), 1-27.

Ahrens, T. \& Mollona, M. (2007). Organizational control as cultural practice -a shop floor ethnography of a Sheffield steel mil. Accounting, Organizations and Society, 32(4-5), 305-331.

Alvesson, M. \& Kärreman, D. Interfaces of control: technocratic and socio-ideological control in a global management consultancy firm. Accounting, Organizations and Society, 29(3-4), 423-444.

Bhimani, A. (2003). A study of the emergence of management accounting system ethos and its influence on perceived system success. Accounting, Organizations and Society, 28(6), 523-548.

Bisbe, J. \& Malagueño, R. (2012). Using strategic performance measurement systems for 
strategy formulation: does it work in dynamic environments? Management Accounting Research, 23, 296-311.

Bisbe, J. \& Otley, D. (2004). The effects of the interactive use of management control systems on product innovation. Accounting, Organizations and Society, 29(8), 709-737.

Boland, R. J., Sharma, A. K. \& Afonso, P. S. (2008). Designing management control in hybrid organizations: the role of path creation and morphogenesis. Accounting, Organizations and Society, 33(7-8), 899-914.

Buehler, K., Freeman, A. \& Hulme, R. (2008b). Owning the right risks. Harvard Business Review, September, 102-110.

Chenhall, R. H. (2003). Management control systems design within its organizational context: findings from contingency-based research and directions for the future. Accounting, Organizations and Society, 28(2-3), 127-168.

Chenhall, R. H. (2008). Accounting for the horizontal organization: a review essay. Accounting, Organizations and Society, 33(4-5), 517-550.

Chenhall, R. H. \& Euske, K. J. (2007). The role of management control systems in planned organizational change: an analysis of two organizations. Accounting, Organizations and Society, 32(7-8), 601-637.

Chua, W. F. (2007). Accounting, measuring, reporting and strategizing - re-using verbs: a review essay. Accounting, Organizations and Society, 32(45), 487-494.

Das, T. K. \& Teng, B. S. (2001). Trust, control, and risk in strategic alliances: an integrated framework. Organization Studies, 22(2), 251-283.

Dávila, T. (2005). An explanatory study on the emergence of management control systems: formalizing human resources in small growing firms. Accounting, Organizations and Society, 30(3), 223-248.
De Harlez, Y. \& Malagueńo, R. (2016). Examining the joint effects of strategic priorities, use of management control systems, and personal background on hospital performance. Management Accounting Research, 30, 2-17.

Dekker, H. C. (2004). Control of inter-organizational relationships: evidence on appropriation concerns and coordination requirements. Accounting, Organizations and Society, 29(1), 27-49.

Dekker, H. C. (2008). Partner selection and governance design in interfirm relationships. Accounting, Organizations and Society, 33(7-8), 915-941.

DiMaggio, P. J. \& Powell, W. W. (1983). The iron cage revisited: institutional isomorphism and collective rationality in organizational fields. American Sociological Review, 48(2), 147-160.

Flamholtz, E., Das, T. \& Tsui, A. (1985). Toward an integrative framework of organizational control. Accounting Organizations and Society, 10(1), $35-50$.

Gray, R. (2002). Of messiness, systems and sustainability: towards a more social and environmental finance and accounting. British Accounting Review, 34(4), 357-386.

Henri, J. F. (2006). Management control systems and strategy: a resource-based perspective. Accounting, Organizations and Society, 31(6), 529-558.

Hopwood, A.G. (1996). Looking across rather than up and down: on the need to explore the lateral processing of information. Accounting, Organizations and Society, 21, 589-590.

Institute of Management Accountants (IMA). (2008). Definition of management accounting. Practice of management accounting. Statements on management accounting. Recuperado de http:// www.imanet.org/docs/default-source/thought_leadership/transforming_the_finance_function/ 
definition_of_management_accounting. pdf?sfvrsn $=2$

Johnson, H. T. \& Kaplan, R. S. (1987). Relevance lost: the rise and fall of management accounting. Boston: Harvard Business School Press.

Kaplan, R. S. (1984). The evolution of management accounting. En Emmanuel, C., Otley, D. y Merchant, K. (Eds.). (1992). Readings in accounting for management control. London: Chapman \& Hall.

Koufteros, X., Verghese, A. J. \& Lucianetti, L. (2014). The effect of performance measurement systems on firm performance: A cross-sectional and a longitudinal study. Journal of Operations Management, 32, 313-336.

Langfield-Smith, K. (1997). Management control systems and strategy: A critical review. Accounting, Organizations and Society, 22(2), 207-232.

Macintosh, N. \& Quattrone, P. (2010). Management accounting and control systems. United Kingdom: Wiley.

Malmi, T. \& Brown, D. A. (2008). Management control systems as a package: opportunities, challenges and research directions. Management Accounting Research, 19, 287-300.

Merchant, K. A. (1985). Control in business organizations. Boston: Pitman.

Metzger, M. B. (2008). The ten commandments of legal risk management. Business Horizons, 51(1), 13-19.

Mills, P. K. (1983). Self-Management: its control and relationship to other organizational properties. Academy of Management Review, 8(3), 445-453.

Mouritsen, J. \& Thrane, S. (2006). Accounting, network complementarities and the development of inter-organizational relations. Accounting, Organizations and Society, 31(3), 241-275.
Norreklit, H. (2000). The balance on the balanced scorecard - a critical analysis of some of its assumptions. Management Accounting Research, 11(1), 65-88.

Norris, G. \& O’Dwyer, B. (2004). Motivating socially responsive decision making: the operation of management controls in a socially responsive organization. The British Accounting Review, 36(2), 173-196

Otley, D. (1994). Management control in contemporary organizations: towards a wider framework. Management Accounting Research, 5, 289-299.

Otley, D. (1999). Performance management: a framework for management control systems research. Management Accounting Research, 10(4), 363-382.

Peters, M. D., Wieder, B., Sutton, S. G. \& Wakefield, J. (2016). Business intelligence systems use in performance measurement capabilities: Implications for enhanced competitive advantage. International Journal of Accounting Information Systems, 21, 1-17.

Porter, M. E. (1980). Competitive strategy. New York: The Free Press.

Power, M. (2007). Organized uncertainty: designing a world of risk management. London: Oxford.

Simons, R. (1999). How risky is your company? Harvard Business Review, 77(3), 85-94.

Wiersma, E. (2008). An exploratory study of relative and incremental information content of two non-financial performance measures: field study evidence on absence frequency and on-time delivery. Accounting, Organizations and Society, 33(23), 249-265.

Wouters, M. \& Wilderom, C. (2008). Developing performance-measurement systems as enabling formalization: a longitudinal field study of a logistics department. Accounting, Organizations and Society, 33(4-5), 488-516. 


\section{Datos de filiación}

\section{Mónica Hernández Madrigal}

Doctora en Metodologías y Líneas de Investigación en Contabilidad y Auditoría por la Universidad de Cantabria, España. Profesora-investigadora de tiempo completo en la Universidad Autónoma de San Luis Potosí en México, en cuya Facultad de Contaduría y Administración imparte las asignaturas de Administración de Costos y Contabilidad. Autora y coautora de artículos sobre contabilidad de gestión. Ha participado como ponente en distintos congresos y reuniones de la especialidad.

Correo electrónico: hmadrigalmonica@uaslp.mx 